\title{
Activation des gènes de l'hémoglobine au cours du développement
}

Plus de travaux ont été consacrés dans le monde aux gènes de globine et au contrôle de leur expression qu'à tout autre gène. C'est qu'ils sont un remarquable modèle de contrôle de l'expression de gènes au cours de la différenciation tissulaire et du développement et qu'ils sont impliqués dans des maladies graves et extrêmement fréquentes. Alors que la séquence nucléotidique complète de ces gènes et des régions bordantes est connue depuis longtemps, l'élucidation des mécanismes de la commutation de l'expression des gènes de globine aux différents stades de la différenciation érythroïde demeure très incomplète: il reste de beaux jours pour les chercheurs étudiant ce système.

\section{Dominique Labie}

Directeur de recherche à l'Inserm. Directeur de l'U.15 de l'Inserm

Rajagopal Krishnamoorthy Chargé de recherche à l'Inserm

\section{ADRESSE}

D. Labie, R. Krishnamoorthy : Inserm U. 15 , CHU Cochin, 24, rue du Faubourg-SaintJacques, 75014 Paris, France.

$m / s n^{\circ} 7$ vol. 4, seplembre 88

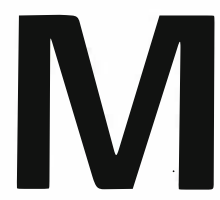

algré de très nombreux travaux concernant la structure, la fonction ou la pathologie moléculaire de l'hémoglobine, on sait encore peu de choses sur la façon dont est contrôlée l'expression séquentielle des différents gènes de globine au cours du développement. Il s'agit pourtant d'un problème d'intérêt majeur, tant du point de vue de la connaissance que dans ses applications pratiques potentielles. La variabilité d'expression de l'hémoglobine joue en effet un rôle important en modifiant l'évolution clinique des grandes hémoglobinopathies. Savoir moduler cette expression en agissant plus ou moins sur un processus évolutif normal serait évidemment un outil incomparable dans ces maladies génétiques à la fois graves et fréquentes.

Nous rappellerons l'organisation normale des gènes de globine chez l'homme ainsi que les variations de leur expression au cours du développement ontogénique. Nous verrons ensuite quelles sont les différentes approches qui ont été utilisées pour essayer de comprendre les mécanismes en cause.

\section{Les étapes du} développement normal

Toutes les hémoglobines sont des tétramères associant deux sous-unités de la série $\alpha$ et deux sous-unités de la série $\beta$. Les deux familles de gènes codant pour ces sous-unités de globine sont disposées, les gènes $\alpha$ sur le bras court du chromosome 16 , les gènes $\beta$ sur le bras court du chromosome 11 , dans les deux cas, de 5 ' en 3', dans l'ordre où ils seront exprimés au cours du développement.

Cette organisation, sur environ $30 \mathrm{~kb}$ (kilobases) pour le complexe $\alpha$ et $60 \mathrm{~kb}$ pour le complexe $\beta$, est résumée dans la figure 1 (page 429). La structure de tous les gènes de globine est similaire, trois exons et deux introns, la principale différence étant que le deuxième intron est beaucoup plus long que le premier dans la famille $\beta$ (intron $l \simeq 120-140 \mathrm{pb}$, intron $2 \simeq 900 \mathrm{pb}$ ) alors qu'ils sont du même ordre de grandeur, moins 


\section{RÉFÉRENCES}

1. Maniatis T, Fritsch EF, Lauer J, Lawn RM. The molecular genetics of human hemoglobins. Annu Rev Genet 1980 ; 14 : 145-78.

2. Collins FS, Weissman SM. The molecular genetics of human hemoglobin. Prog Nucleic Acid Res Mol Biol 1984 ; 31 : 315-438.

3. Liebhaber SA, Goossens M, Kan YW Homology and concerted evolution at the alpha 1 and alpha 2 loci of human alphaglobin. Nature 1981 ; 290 : 26-9.

4. Michelson A, Orkin SH. Boundaries of gene conversion within the duplicated human $\alpha$-globin genes. J Biol Chem 1983; 258: $15245-9$

5. Slightom JL, Blechl AE, Smithies O. Human fetal G gamma and A gamma globin genes : complete nucleotide sequences suggest that DNA can be exchanged between these duplicated genes. Cell 1980; 21 : 627-8.

6. Smithies O, Engels WR, Devereux JR, Slightom JL, Shen S. Base substitutions, length differences and DNA strand asymetries in the human $\mathrm{G}$ gamma and A gamma fetal globin gene region. Cell 1981 ; 26 : 345-53.

7. Bunn HF, Forget BG. Hemoglobin : molecular genetic and clinical aspects. Philadelphia: WB Saunders, 1986: 61-8.

8. Karlsson S, Nienhuis AW. Developmental regulation of human globin genes. Ann Rev Biochem 1985; 54 : 1071-108.

9. Schroeder WA, Huisman TH, Shelton R, et al. Evidence for multiple structural genes for the gamma chain of fetal human hemoglobin. Proc Natl Acad Sci USA 1968; 60 : 537-44.

10. Labie D, Dunda-Belkhodja O, Rouabhi F, Pagnier J, Ragusa A, Nagel RL. The -158 site 5' to the $G_{\gamma}$ gene and $G_{\gamma}$ expression. Blood de $150 \mathrm{pb}$ (paires de bases), dans la famille $\alpha[1,2]$.

On remarquera que plusieurs de ces gènes sont le résultat de duplications. Ceci est particulièrement évident en ce qui concerne les gènes $\alpha_{2}$ et $\alpha_{1}$, dont les produits d'expression protéique sont identiques, et aussi les gènes $G \gamma$ et $A \gamma$ dont les produits ne diffèrent que par un acide aminé en position 136 (glycocolle et alanine). La conservation de cette identité reflète un processus de conversion réciproque au cours de l'évolution [3-6].

Il faut rappeler l'existence, parce que nous en reverrons le rôle possible, de séquences répétitives de type $A l u$ et de type $K p n$, encadrant les différentes régions du complexe $\beta$, dont les motifs homologues sont répartis ailleurs dans le génome humain. Il y a en particulier deux séquences $A l u$ en position inversée en amont du gène $\delta[2]$.

A $\ddot{u}$ cours du développement, on observe des changements, tant au niveau des organes où sont synthétisées les globines, que dans la nature de celles-ci. Ces changements sont à peu près synchrones sans cependant qu'on puisse identifier un type de sous-unités à un lieu de synthèse. Des cohortes cellulaires successives essaimant des différents lieux de synthèse expriment majoritairement l'une ou l'autre hémoglobine, embryonnaire, fœtale ou adulte. Mais ce phénomène n'est jamais exclusif; à toute étape de la vie on peut identifier simultanément plusieurs hémoglobines. Les globines embryonnaires, $\zeta$ et $\varepsilon$, sont synthétisées très précocément, essentiellement au niveau du sac vitellin. Elles sont progressivement remplacées, entre la $6^{\mathrm{e}}$ et la $8^{\mathrm{e}}$ semaine, par les globines de la vie fœtale, selon les deux commutations :

$$
\zeta \rightarrow \alpha_{1} \text { et } \alpha_{2} \text { et } \varepsilon \rightarrow \mathrm{G} \gamma \text { et } \mathrm{A} \gamma
$$

la commutation $\zeta \rightarrow \alpha$ étant légèrement plus précoce que la commutation $\varepsilon \rightarrow \gamma[7,8]$. Pendant le reste de la vie foetale, le foie et la rate sont les sources majeures de la production des globules rouges, bien que l'activité de la moelle osseuse commence dès le second trimestre. Elle deviendra majoritaire à la fin de la vie foetale. Une érythropoïèse extramédullaire peut réapparaître dans des conditions pathologiques [7, 8].

Les gènes $G \gamma$ et $A \gamma$ sont exprimés selon un rapport à peu près constant pendant la vie foetale: $\mathrm{G} \gamma / \mathrm{A} \gamma$ environ $3 / 1$. Cependant, dans le cas du complexe $\beta$, la synthèse de chaîne $\beta$, qui s'est maintenue faible pendant plusieurs mois, subit une augmentation brusque un peu avant la naissance. Elle représenterait à ce moment environ la moitié de la synthèse des chaînes non $\alpha$. La synthèse de la chaine $\delta$, plus tardive, restera toujours faible. Parallèlement, mais non simultanément, il y a modification du rapport d'expression des deux chaînes $\gamma$, le rapport $\mathrm{G} \gamma / \mathrm{A} \gamma$ passant classiquement de $3 / 1$ à environ 2/3 [9]. Ces chiffres, on le sait par des études de séries plus longues, sont très schématiques. Ils donnent cependant dans la majorité des cas un ordre de grandeur. L'existence d'une évolution est quasiment constante, les valeurs individuelles sont génétiquement déterminées, en rapport avec la structure du locus $\gamma[10,11]$.

Ce sont ces deux commutations $(\mathrm{G} \gamma$ et $A \gamma \rightarrow \delta$ et $\beta ; G \gamma / A \gamma$ de mode fotal $\rightarrow \mathrm{G} \gamma / \mathrm{A} \gamma$ de mode adulte), qui ont fait l'objet de nombreux travaux. Ce switch se traduit simultanément dans tous les organes hématopoïétiques. Il est en rapport étroit avec l'âge gestationnel, et contemporain dans la lignée érythrocytaire d'autres commutations touchant par exemple les isozymes de l'anhydrase carbonique ou des antigènes de surface.

L'évolution se fait après la naissance entre 6 à 12 mois vers un "profil » adulte de la synthèse des chaînes de globine. L'hémoglobine fœtale, normalement inférieure à $1 \%$, se trouve apparemment confinée dans une population restreinte de cellules adultes que l'on appelle les cellules F[12]. Leur pourcentage est génétiquement déterminé, mais il ne s'agit pas d'une population clonale. C'est en fait un seuil quantitatif que l'on détecte, variable selon la méthode employée (environ 5 pg par cellule par élution acide, l pg par immunofluorescence) (pg = picogramme), et il ne serait pas impossible que la restriction observée de la synthèse de chaînes $\gamma$ à une souspopulation de cellules soit en fait un artefact méthodologique [13, 14].

On a cherché dans la structure des 


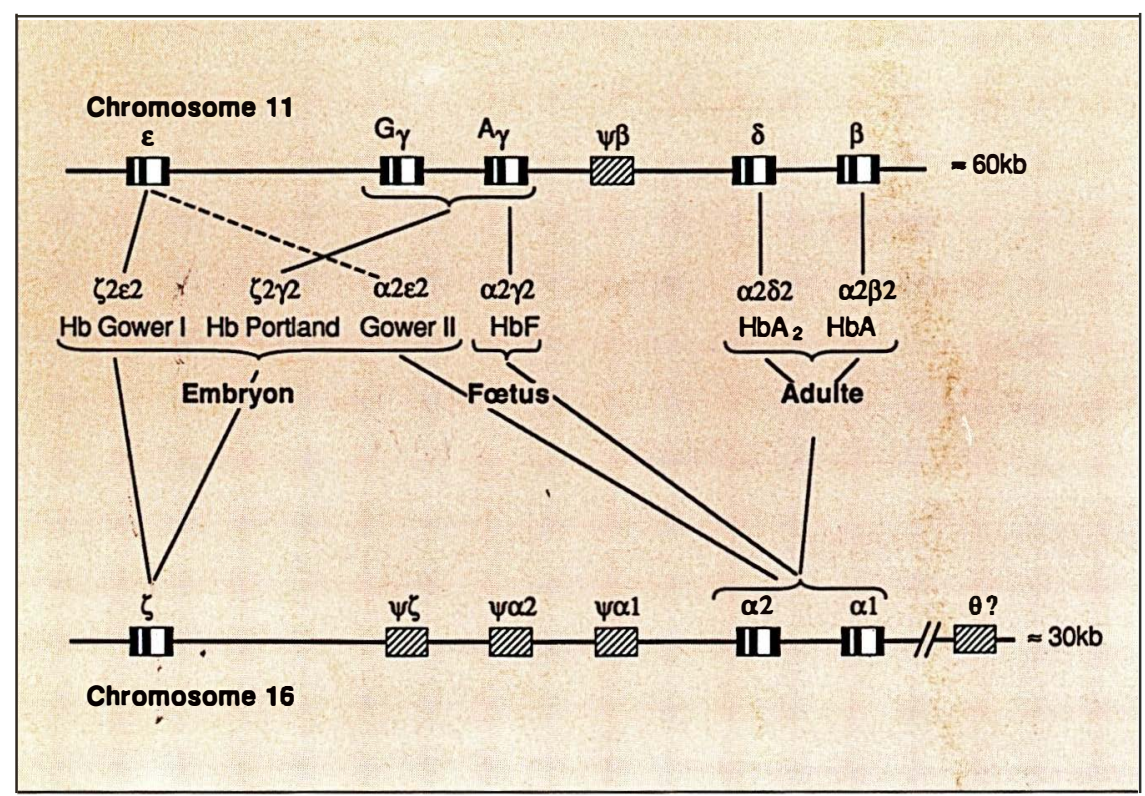

gènes de globine l'explication de leur activation séquentielle. Le développement ontogénique ne s'accompagne d'aucun réarrangement majeur des gènes de globine. Au niveau chromatinien, l'expression d'un gène s'accompagne d'un certain niveau d'hypométhylation et d'une sensibilité accrue à la digestion par des nucléases non spécifiques, mais ces modifications reflètent l'activité d'un gène, elles n'en sont pas la cause [15, 16]. D'autres sites hypersensibles, entourant de plus loin le complexe génomique $\beta$, reflètent une structure particulière de la chromatine spécifique des tissus érythropoïétiques [17]. Quant aux études de séquence, elles ne donnent encore que des indices d'interprétation difficile et incomplète.

On peut schématiquement dire que l'étude des mécanismes impliqués a suscité deux types d'abord. Le premier est l'observation de modifications naturelles, le plus souvent pathologiques, le deuxième est l'utilisation d'un certain nombre de modèles expérimentaux.

\section{Les dérégulations physiopathologiques}

Affections dont le phénomène majeur est l'augmentation de l'hémoglobine F. Plus de 50 mutations $\mathrm{m} / \mathrm{s} n^{\circ} 7$ vol. 4 , seplembre 88 ont actuellement été décrites et analysées dont la conséquence majeure est une persistance héréditaire d'hémoglobine foetale (PHHF). La description en a d'abord été phénotypique et d'excellentes revues existent [7, 13]. Sur le plan hématologique, une PHHF peut être pancellulaire ou hétérocellulaire, elle peut s'accompagner d'une hypochromie microcytaire ou être morphologiquement normale. Ces descriptions initiales mettaient donc en évidence polymorphisme et complexité. L'étude de l'hémoglobine fœutale elle-même a montré l'existence de formes à prédominance $\mathrm{G} \gamma$, de formes $\mathrm{A} \gamma$ ou de formes mixtes. Enfin l'étude de l'ADN a montré qu'il existe des formes délétionnelles et des formes non délétionnelles.

- Les mutations délétionnelles sont de longueur et de localisation variables, que seule une figure peut essayer de résumer (figure 2, page 430) [7]. Sur le plan physiopathologique, elles réalisent un spectre très varié de désordres dont les points communs sont: l'absence complète de synthèse $\beta$ (éventuellement aussi $\delta$ et $A \gamma$ ), et la compensation plus ou moins complète par une synthèse de chaîne $\gamma$.

Le nom - PHHF ou $\delta \beta$ thalassémie - est fonction de la gravité plus ou moins grande de la maladie, mais

Figure 1. Schéma du contrôle génétique des hémoglobines humaines au cours du développement. La période embryonnaire se termine entre la $6^{e}$ et la $8^{e}$ semaine. Le profil foetal est observé à partir de cette date. C'est entre la $28^{e}$ et la $32^{e}$ semaine que la synthèse des chaînes $\gamma$ diminue progressivement, et que celle des chaînes $\beta$ augmente. A la naissance, il y a encore une large prédominance de I'HbF, qui diminue rapidement, puis plus lentement, pour atteindre un profil adulte en six mois à un an. Les causes de cette variabilité chronologique périet post-natale, sans doute génétiques, sont encore mal connues. Les exons sont représentés par des rectangles noirs et les introns par des rectangles blancs. Les pseudogènes sont symbolisés par des rectangles hachurés.

il y a toujours augmentation absolue de la synthèse $\gamma$ qu'on est tenté de rattacher à la délétion [18]. Plusieurs hypothèses ont pu être formulées: (1) Une zone «régulatrice» existerait en amont du gène $\delta$, au niveau des séquences $A l u$. L'inclusion, ou non, de ces séquences dans la partie délétée expliquerait le niveau de synthèse de l'hémoglobine $F$, et donc le phénotype. Ce schéma n'a pu s'appliquer qu'à un nombre très limité de cas. (2) Dans un autre schéma, l'attention s'est focalisée, non plus sur la partie délétée, mais sur les séquences en cis que le phénomène délétionnel rapproche des gènes $\gamma$. Des séquences enhancer semblables à des séquences virales ont pu être caractérisées. Ce schéma a semblé valable pour quelques délétions de grande taille. Cependant, même dans ce cas, on voit, sur la figure 2, que l'extrémité 3' de toutes ces délétions est différente : il semble peu probable que toutes ces extrémités contiennent, à répétition, des séquences enhancer. (3) Une troisième hypothèse, la plus séduisante peut-être, est celle de domaines chromatiniens, fotal et adulte, successivement activés. La destruction des frontières perturberait cette activation séquentielle. A l'appui de ce schéma, deux faits: (a) l'existence d'une polarité semble établie par plusieurs observa- 


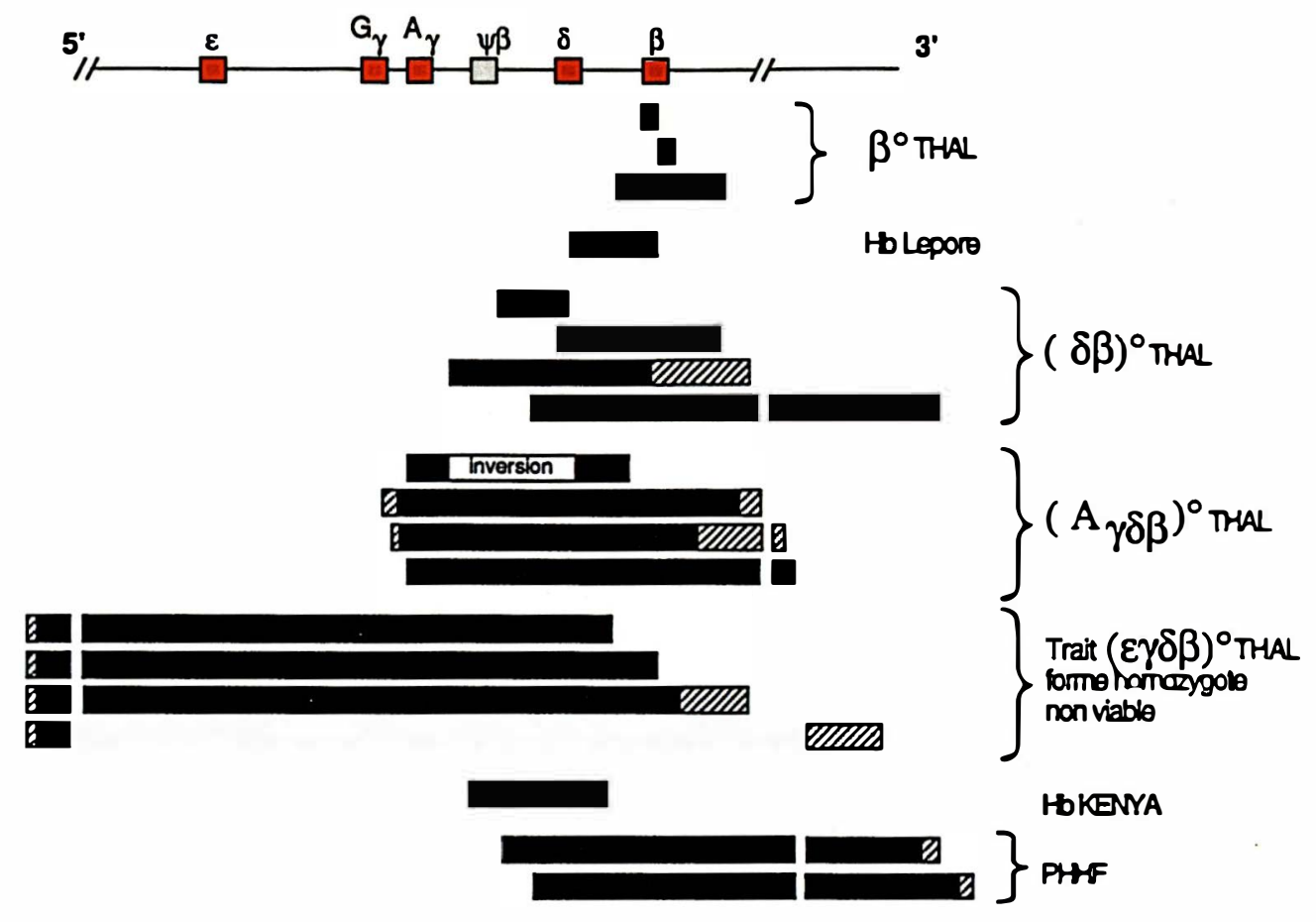

Figure 2. Figuration (incomplète) des diverses délétions décrites dans le complexe génomique $\beta$. Elles ont été groupées selon le mode de phénotype qui en est la conséquence. $\beta^{\circ}$ Thal = Thalassémie sans expression de la chaîne $\beta$; Hb Lepore = gène de fusion $\delta \beta$. Le produit d'expression est une protéine hybride exprimée à environ $10 \%$ chez l'hétérozygote, donc thalassémique; $(\delta \beta)^{\circ}$ Thal = syndrome thalassémique, généralement modéré, dans lequel ne sont exprimées ni la chaîne $\beta$, ni la chaîne $\delta ;(A \gamma \delta \beta)^{\circ}$ Thalassémie = syndrome thalassémique, dans lequel la chaîne Gr seule est exprimée; $(\varepsilon \gamma \delta \beta)^{\circ}$ Thalassémie = sur le chromosome atteint, aucune des chaînes du complexe génomique $\beta$ n'est exprimée, seules des formes hétérozygotes sont observées; Hb Kenya = gène de fusion A $\gamma$ - $\beta$ et protéine hybride; PHHF (persistance héréditaire de l'hémoglobine foetale) délétionnelle = syndrome où ne sont exprimées ni chaîne $\beta$ ni chaîne $\delta$, mais où du fait d'une synthèse de chaîne $\gamma$ plus élevée que dans les $(\delta \beta)^{\circ}$ thalassémies, la condition est mieux tolérée et presque inapparente. II faut noter qu'il n'y a pas de frontière précise entre ces deux séries pathologiques. En traits pleins, les zones délétées; en traits hachurés, les zones des extrémités sur lesquelles persistent des imprécisions. Les interruptions (au niveau du chromosome et des délétions) correspondent aux délétions trop grandes pour être figurées à l'échelle.

tions dans lesquelles une délétion inhibe l'expression d'un gène situé en aval, et cependant présent [19]. (b) Par ailleurs, le fait que différentes délétions s'étendent sur environ $100 \mathrm{~kb}$ suggère un mécanisme qui pourrait être la disparition complète d'une boucle de chromatine entre deux points d'attache au stroma [20].

Il reste qu'aucun modèle ne semble pouvoir être général, et que la question se pose de la mesure dans laquelle ces formes perturbées sont susceptibles d'éclairer un fonctionnement normal.

- Les formes non délétionnelles, dans lesquelles une synthèse de compagnent en général d'aucun trouble, tirent leur intérêt potentiel de ce qu'elles sont plus proches d'un modèle physiologique. Elles sont également hétérogènes dans leur expression. Certaines sont liées à des mutations des régions promotrices $\gamma$. Elles sont résumées dans la figure 3 . Ces mutations ponctuelles, groupées sur une centaine de paires de bases, en amont des séquences liant la polymérase, s'accompagnent en règle générale d'une augmentation spécifique du gène qui suit la mutation soit $G \gamma$, soit A $\gamma$. Il est possible qu'elles modifient des interactions ADN/protéines impliquées dans la régulation au cours du développement [12]. A l'appui de cette hypo- thèse, on peut remarquer que dans cette zone «à risque » où sont groupées la majorité des mutations, on retrouve également plusieurs «motifs » homologues de séquences connues dans d'autres systèmes pour lier des protéines régulatrices d'expression*.

Il existe enfin quelques formes, dont la base moléculaire est pour l'instant totalement inconnue, dont les études familiales font penser qu'elles

\footnotetext{
* Depuis la rédaction de cet article, un cas a été publié dans lequel la PHHF semble en rapport avec une forme variante de la séquence

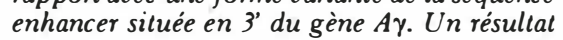
similaire a simultanément été retrouvé dans le laboratoire des auteurs de cet article.
} 


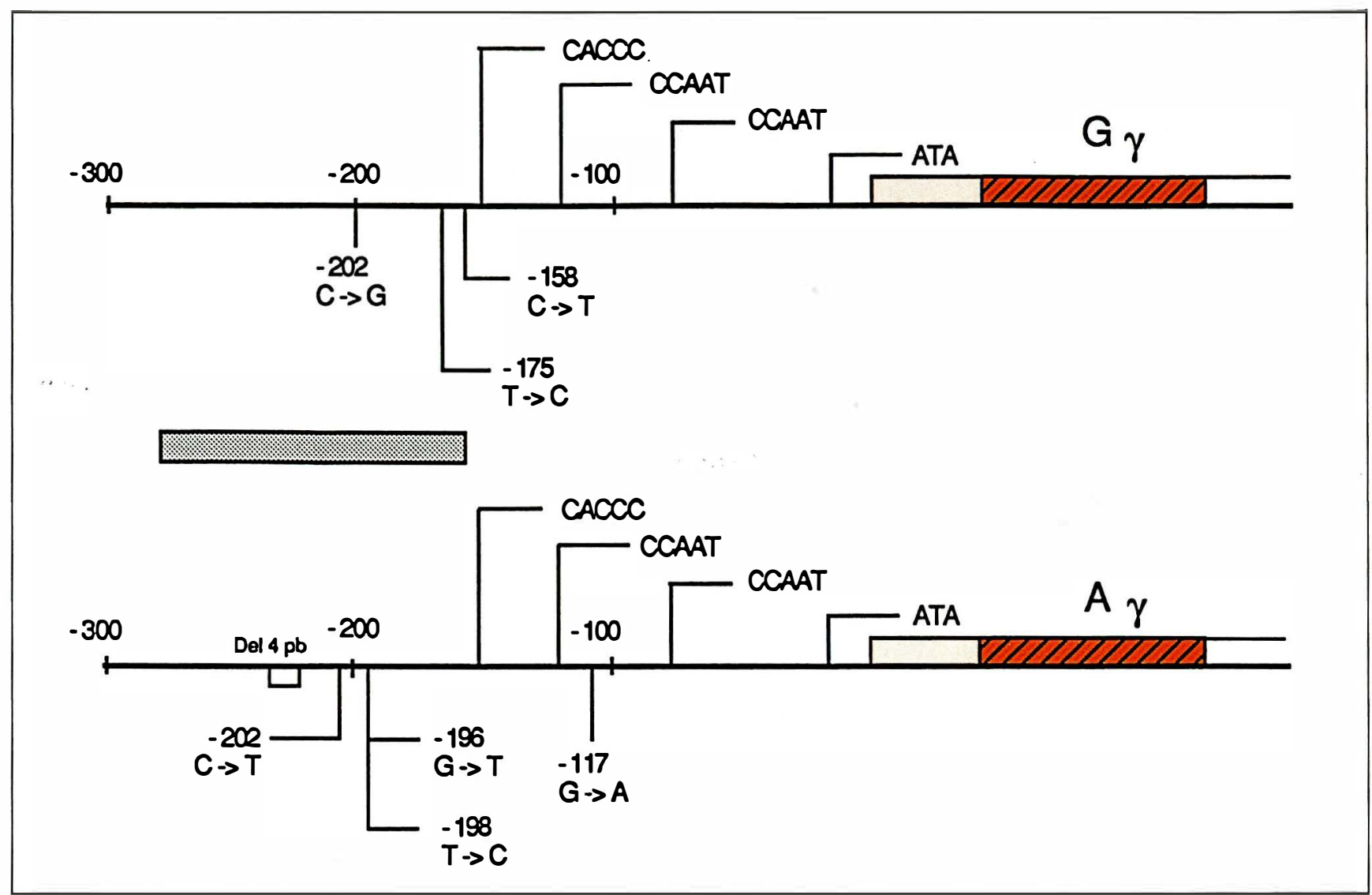

Figure 3. Zone promotrice des gènes G $\gamma$ et A $\gamma$. En amont du site d'initiation se trouvent d'abord les séquences nécessaires à la transcription et communes à beaucoup de gènes eucaryotes (TATA box, CCAAT box, CACCC box); puis une zone (rectangle pointillé) où sont regroupées des séquences plus ou moins homologues de "motifs „ décrits dans d'autres systèmes (SV 40 , immunoglobulines, etc.). Dans ces systèmes on a démontré qu'elles sont le site de liaison de facteurs de régulation agissant en trans. On a figuré les différentes mutations qui se regroupent toutes dans ces zones, ou à leur frontière. En l'absence de données expérimentales claires démontrant l'action régulatrice de protéines spécifiques à ce niveau, le fait que diverses mutations ponctuelles augmentent l'expression du gène situé en aval est un argument indirect mais fort pour attribuer à cette zone un rôle critique de régulation au cours du développement. Rectangles rouges = partie codante des exons; rectangles roses $=$ partie non codante des exons $;$ rectangles blancs $=$ introns.

ne seraient pas liées au locus $\beta$. Augmentation de l'hémoglobine $F$ dans d'autres maladies hématologiques génétiques. A côté des mutations directement responsables d'une synthèse anormale d'hémoglobine $F$ dans la vie adulte, d'autres maladies hématologiques congénitales s'accompagnent d'une augmentation de l'hémoglobine $\mathrm{F}$ apparemment secondaire. Il s'agit essentiellement de deux affections majeures, la drépanocytose et les $\beta$ thalassémies. Dans un cas comme dans l'autre, le mécanisme d'augmentation de l'hémoglobine $F$ est complexe, dû en partie à une survie sélective des cellules $\mathrm{F}$ : parce que l'hémoglobine $\mathrm{F}$ ne copolymérise pas avec l'hémoglo- bine $S$ et empêche la transformation falciforme des cellules dans le premier cas : parce que l'hémoglobine $F$, en s'associant aux chaînes $\alpha$ en excès, empêche la destruction prématurée des cellules dans le deuxième. Mais, à côté de ce mécanisme physiopathologique, il existe aussi une augmentation vraie de la synthèse des chaînes $\gamma$. Celle-ci varie qualitativement et quantitativement selon les haplotypes de restriction des chromosomes porteurs de la mutation pathogène, ceci quelle que soit la mutation, $\beta^{\mathrm{s}}$ ou $\beta^{\text {Thal }}[10]$. Elle semble donc due à la présence de déterminant(s) génétique(s) spécifique(s). A ces deux affections les plus fréquentes, il faut ajouter deux types d'anémie congénitale aplastique : la maladie de Blackfan-Diamond et la maladie de Fanconi. On y observe régulièrement une augmentation de l'hémoglobine $\mathrm{F}$.

Augmentations acquises de l'hémoglobine fotale. Enfin l'augmentation de l'hémoglobine F peut être un phénomène acquis. Elle est classique au cours de tout stress érythropoiétique aigu qui s'accompagne d'une régénération médullaire rapide, mais également quand il y a une expansion de l'érythropoïèse, après une hémolyse aiguë par exemple, ou au cours du deuxième trimestre de la grossesse. Il s'agit alors d'une accélération des étapes de la différenciation. 
L'augmentation périphérique de l'hémoglobine $\mathrm{F}$ est aussi un signe de nombreuses maladies hématopoḯtiques malignes. Elle est presque constante dans la leucémie myéloïde juvénile chronique, accompagnée d'autres caractéristiques fotales, mais elle est également observée au cours d'autres désordres myéloprolifératifs. La base moléculaire est encore mal connue, il semble y avoir distorsion d'une régulation coordonnée plutôt que retour à une érythropoïèse fœtale vraie [18].

\section{Abords expérimentaux}

A côté de cette analyse de phénomènes spontanés, diverses approches expérimentales ont été utilisées pour étudier le phénomène du switch. Ces modèles expérimentaux sont discutés tous les deux ans lors d'une conférence spécialisée dont le compte rendu est publié régulièrement. Les deux derniers volumes sont particulièrement riches [21, 22].

Transplantation de cellules hématopoïétiques. On a utilisé le fait que les étapes du développement dans la synthèse de l'hémoglobine sont similaires chez les ovins et chez l'homme pour réaliser chez les premiers des expériences de transplantation des cellules hématopoḯtiques: l'implantation de cellules foetales chez des agneaux nouveau-nés irradiés a permis de discriminer entre le rôle de l'environnement, qui aurait pu provoquer une commutation immédiate des cellules implantées, et celui d'un mécanisme intrinsèque à cette même cellule, une horloge biologique de développement. C'est ce qu'on observe : la cellule implantée continue à synthétiser de l'hémoglobine fœetale, la transition observée est en rapport avec l'âge de gestation de l'animal donneur et non de l'animal receveur [23].

Expression des gènes dans des lignées néoplasiques. Un certain nombre de lignées ont actuellement été établies à partir de cellules leucémiques, qui peuvent se différencier en cellules érythroïdes après induction. Il y a eu d'abord la lignée MEL de l'érythroleucémie de souris qui, quand on induit sa différenciation érythroïde, produit une hémoglobine adulte. Il existe aussi des lignées humaines. La lignée $K 562$, dérivée d'un sujet porteur d'une leucémie myéloïde, produit des chaînes embryonnaires et des chaînes fotales en proportion moindre. Le gène $\beta$ n'y est jamais exprimé, bien qu'il soit présent et qu'on puisse, après clonage, le faire exprimer dans un autre environnement cellulaire. De même, la lignée HEL n'exprime que des hémoglobines fœtales. Tout se passe comme si ces lignées, originaires de sujets adultes, avaient été gelées à des stades précoces du développement. Elles ont été utilisées pour des expériences de transfert et la recherche de facteurs régulateurs trans* [13].

Transfert de chromosomes ou de gènes. Les gènes de globine ont pu être introduits dans les cellules MEL qui sont de type «adulte», soit sous forme de chromosome intact, par fusion cellulaire réalisant des hybrides somatiques, soit sous la forme de gènes clonés. Dans le premier cas il y a, sous l'action d'un inducteur, et parallèlement à l'expression du gène endogène, expression du gène adulte correspondant au chromosome transféré $(\beta$ dans le cas du chromosome $11, \alpha$ dans celui du chromosome 16) à l'exclusion des gènes embryonnaires et fœetaux portés par le même chromosome [24].

L'introduction de gènes clonés dans des cellules MEL donne également lieu à une expression sélective des gènes adultes, même quand ces gènes sont originaires de cellules où ils ne s'exprimaient pas, telles les cellules K 562 ou HEL. A l'inverse, dans des cellules "gelées» à l'état fotal - K 562 par exemple - on ne peut faire exprimer que des gènes embryonnaires ou foetaux, à l'exclusion de gènes adultes. Ceci est interprété comme la démonstration de la présence dans ces cellules de facteurs trans, spécifiques d'une étape du développement, et interagissant au moment de la transcription avec les séquences présentes en cis des gènes transférés.

La localisation de ces séquences présentes en cis s'est faite grâce à la construction de gènes chimériques.

\footnotetext{
* Facteur trans, ou facteur actif en trans: facteur diffusible modulant, à distance, l'activilé d'un gène.
}

\section{construction de gènes chimériques.} mia syndromes. Oxford : Blackwell Scienti publications, 1981 : 450-507.

17. Tuan D, Solomon W, LI Q London IM. erythoid cells. Proc Natl Acad. Sci USA 1985 ; 
Des promoteurs, explorés jusqu'à 300 ou 400 nucléotides en amont du site d'initiation de la transcription ont été liés dans diverses constructions aux zones codantes d'autres gènes. On a ainsi pu démontrer qu'il existe dans les régions promotrices des séquences spécifiques des étapes du développement : seuls les promoteurs $\varepsilon$ et $\gamma$ sont fonctionnels dans la lignée K 562, alors que le promoteur $\beta$ fonctionne mieux que le promoteur $\gamma$ dans les cellules MEL. Dans ce type d'études des informations complémentaires ont été obtenues en utilisant les mutants PHHF non délétionnels: une substitution en -117 du gène $A \gamma$, un groupe de substitutions autour de -200 de l'un ou l'autre des gènes $\gamma$, et à un moindre degré une substitution en -158 du gène $G \gamma$, semblent marquer des lieux d'interaction entre les promoteurs et les facteurs trans susceptibles de moduler l'expression des gènes $\gamma$. Des délétions expérimentales semblent par ailleurs montrer qu'il existe, en un site encore plus distal, une autre zone d'effet positif. D'autres constructions ont également mis en évidence la présence de séquences régulatrices dans les régions codantes, pour l'expression des gènes $\gamma$, en particulier au niveau du deuxième intron.

Expression dans des souris transgéniques. Par injection dans le noyau d'œufs de souris fertilisés, on peut obtenir l'intégration stable d'éléments génétiques étrangers, transmissibles. Le terme de « souris transgénique » désigne les animaux qui, ayant survécu, expriment en se développant les gènes ainsi intégrés. De telles expériences ont été employées pour déterminer les séquences en cis du gène intégré nécessaires pour qu'il soit exprimé. On a pu mettre en évidence, à proximité des gènes, des séquences conférant la spécificité tissulaire d'expression - un gène de globine n'est exprimé qu'en tissu érythroïde - mais aussi des séquences conférant la spécificité d'expression à un stade donné du développement. Un gène fotal est exprimé dans le tissu fœetal, un gène adulte dans le tissu adulte, et de façon concomitante aux gènes endogènes de la souris. Cependant, si on n'injecte qu'un gène et les séquences immédiatement avoisinantes, l'ex-

$\mathrm{m} / \mathrm{s} n^{\circ} 7$ vol. 4 , seplembre 88 pression est correctement contrôlée, mais elle reste aléatoire et faible* Des expériences plus récentes ont montré le rôle de l'injection simultanée de séquences agissant également en cis, mais situées à distance du gène que l'on veut exprimer. De telles séquences dont l'action stimulatrice répond à la définition d'un enhancer: action à distance, indépendante de l'orientation et de la position, ont été mises en évidence en aval du gène $A \gamma$ et en aval du gène $\beta^{*}[25,26]$. Plus récemment, enfin, on a pu augmenter, dans des souris transgéniques, le niveau d'expression d'un gène étranger - la $\beta$ globine humaine - jusqu'au niveau du gène endogène, en reconstruisant un locus où ce gène est encadré par des séquences qui sont normalement situées aux deux extrémités du complexe $\beta$ [27]. Ces séquences, qui interagissent probablement avec des facteurs trans spécifiques de tissus, jouent certainement un rôle majeur dans l'expression des gènes de globine.

Expression dans des systèmes de colonies in vitro. L'étude de la capacité des progéniteurs à former des colonies, a donné lieu à une multitude de travaux. De façon générale, on peut dire que l'expression des gènes de globine adulte et fotale dans ces colonies reflète toujours le stade de développement des individus chez qui le prélèvement a été fait : si ces colonies proviennent d'un fœetus, elles produisent surtout des chaînes $\gamma$; si c'est d'un adulte, les chaînes $\beta$ prédomineront. La proportion de chaînes $\gamma$ est cependant toujours supérieure à ce qu'elle était in vivo. En fait, les deux types de chaînes sont toujours produites dans toutes les colonies, et seule leur proportion respective varie selon un continuum étendu. On a cherché à manipuler cette proportion par diverses conditions expérimentales. Les chaînes $\gamma$ apparaissant avant les chaînes $\beta$, on a tenté de modifier les conditions de croissance qui influeraient sur la maturation. Aucun argument ne permet cependant d'extrapoler à la régulation in vivo ces

* Voir nouvelle m/s, $n^{\circ} 4$, vol. 4, p. 252. observations faites en abrégeant ou allongeant la maturation in vitro.

Une observation intéressante est cependant que, dans les cultures produisant des chaînes $\gamma$, et même dans celles dont les progéniteurs provenaient de sujets porteurs d'une PHHF délétionnelle, on peut «éteindre » cette production par un facteur présent dans le sérum de veau fotal. Ces cellules progénitrices étant incapables de produire des chaînes $\beta$, il y aura seulement une chute du rapport de synthèse $\gamma / \alpha$. Tout se passe comme si, aux dernières étapes du développement fœtal, il y avait expression de récepteurs pour un facteur inhibiteur présomptif. Quant à la différenciation vers l'expression fotale ou adulte des cellules au sein d'une même colonie mixte, elle semble relever d'événements stochastiques aux premières étapes de la différenciation, de base moléculaire totalement inconnue, et qu'on ne peut, là encore, pas extrapoler à la régulation in vivo.

Modifications expérimentales de l'expression des gènes $\gamma$ in vivo. Quelques mots, enfin, doivent être dits d'essais faits initialement chez le babouin. Se basant sur le fait qu'un gène exprimé est souvent déméthylé, on a administré de la 5-azacytidine, qui est un agent hypométhylant, et on a observé chez l'animal une augmentation de la production de l'hémoglobine fotale.

Des essais limités ont été faits chez quelques sujets thalassémiques ou drépanocytaires, chez qui une augmentation de synthèse de l'hémoglobine $F$ avait un intérêt potentiel évident. Des résultats inégaux ont été obtenus, ils sont cependant indubitables dans quelques cas [28]. La relation de cause à effet avec l'hypométhylation a cependant été remise en question, car la 5-azacytidine intervient également dans le cycle cellulaire. Des résultats similaires ont également été obtenus avec des agents cytotoxiques mais non hypométhylants, tels que l'hydroxyurée. Une interférence avec la maturation érythroïde a été invoquée, aussi bien qu'une augmentation vraie de l'expression des gènes $\gamma$. Il est possible que les deux mécanismes interviennent dans des proportions variées, et que le second - augmentation de 


\section{RÉFÉRENCES}

19. Bernards R, Flavell RA. Physical mapping of the globin gene deletion in hereditary persistence of fetal hemoglobin (HPFH). Nucleic Acids Res 1980 ; 8 : 1521-34.

20. Collins SF, Cole JL, Lockwood WK, Iannuzzi MC. The deletion in both common types of hereditary persistence of fetal hemoglobin is approximately 105 kilobases. Blood $1987 ; 70$ : 1797-803.

21. Stamatoyannopoulos G, Nienhuis AW Experimental approaches for the study of hemoglobin switching. Progress in Clinical and Biological Research. New York: Alan R Liss Inc, 1985 : 191.

22. Stamatoyannopoulos $\mathrm{G}$, Nienhuis AW Developmental control of globin gene expres sion. Progress in Clinical and Biological Research. New York: Alan R Liss Inc, 1987 251

23. Wood WG, Bunch C, Kelly S, Gunn Y, Breckon G. Control of hemoglobin switching by a developmental clock? Nature $1985 ; 313$ : $320-3$

24. Melis M, Demopulos G, Najfeld V, et al. A chromosome ll-linked determinan controls fetal globin expression and the fetal to adult globin switch. Proc Natl Acad Sci USA 1987; 84 : 8105-9.

25. Bodine DM, Ley TJ. An enhancer element lies 3' to the human A $\gamma$ globin gene EMBO J 1987 ; 6 : 2997-3004.

26. Trudel M, Constantini F. A 3' enhancer element contributes to the stage specific expression of the human $\beta$ globin gene Genes Development 1987 ; 1 : 954-61.

27. Grosveld F, Assendelfı GBV, Greaves DR Kollias G. Position independant, high-level expression of the human $\beta$ globin gene in transgenic mice. Cell $1987 ; 51$ : 975-85.

28. Charache S, Dover G, Smith K, Talbot CC, Mayer M, Boyer S. Treatment of sickle cell anemia with 5-azacytidine results in increased fetal hemoglobin production and is associated with non random hypomethylation of DNA around the $\gamma-\delta-\beta$ globin gene complex. Proc Nall Acad Sci USA 1983; 80 4842-6.

29. AI-Khatti A, Veith RW, Papayannopoulou T, Fritsch EF, Goldwasser E, Stamatoyan nopoulos G. Stimulation of fetal hemoglobin synthesis by erythropoietin in baboons. $N$ l'expression des gènes $\gamma-$ soit fonction d'un terrain génétique, dont on ne connaît pas encore la nature, mais que pourraient refléter les haplotypes de restriction.

Plus récemment enfin, il a été suggéré, et montré, que l'érythropoïétine, administrée en pulse, augmente progressivement la proportion de cellules $\mathrm{F}$, puis la quantité d'hémoglobine F circulante [29].

Ces manipulations pharmacologiques sont actuellement considérées comme la base potentielle du traitement des hémoglobinopathies majeures, puisque la présence d'hémoglobine $\mathrm{F}$ améliore réellement les malades.

\section{Conclusion}

Au terme de cet exposé, il est clair qu'aucun modèle cohérent ne peut être proposé qui soit la synthèse des multiples informations. Une difficulté majeure reste l'incertitude absolue où l'on est de savoir si les modèles pathologiques ou expérimentaux sont transférables à l'analyse d'un développement normal.

Les explications anatomiques restent évidemment insuffisantes. Des lignes de recherche semblent se dessiner au niveau moléculaire dans la mise en évidence de facteurs trans spécifiques des différentes étapes du développement, ainsi que dans le rôle des mutations en cis des régions promotrices pour localiser des zones d'interaction ADN/protéines. Mais des étapes entières concernant notamment la médiation des signaux restent totalement obscures.

Un abord multiple sera sans doute nécessaire, étudiant les étapes de la différenciation cellulaire et le développement de l'individu en même temps que les données de la biologie moléculaire. Cette étude reste difficile du fait qu'il s'agit d'une population cellulaire en renouvellement constant, que les événements moléculaires étudiés sont inaccessibles directement, car ils se produisent dans des progéniteurs qu'on ne connaît que par leur capacité à former des colonies. Par ailleurs, les événements de commutation génique de l'hémoglobine se produisent dans l'organisme à des périodes de changements énormes et multiples. L'abord ne peut donc en être qu'indirect

\section{Summary}

The molecular and cellular mechanisms involved in the sequential expression of human globin genes during development (collectively known as «hemoglobin switching ») have been intensively studied in recent years. It is a pioneer model as well in understanding the regulation of expression in a multigene family as in molecular pathology. The hemoglobinopathies are the most frequent group of inherited disorders varying from the very severe clinical conditions to asymptomatic cases. We first review the changes in expression of globin genes during normal development with an emphasis on the fetal to adult $\mathrm{Hb}$ gene switching, because of its potential therapeutical value. Then we describe how the pathophysiological and experimental models have been useful to get insight into the molecular mechanisms of the switching. The present status of research gives some clues. Some sequences in cis in the promoter area, in the coding regions and in putative enhancer sequences appear to be important in the developmental regulation; sequences further at the limits of the $\beta$ locus allow the definition of tissue specificity. The existence of both tissue and developmental specific trans acting factors have been demonstrated by two different approaches. Despite the intensive research outlined here, many steps remain a mystery. Only a multidisciplinary research including molecular, cellular and genetic aspects of regulation could bring converging data for explaining the switching mechanisms.

\section{TIRÉS A PART}

D. Labie: Inserm I'. 15, CHL Cochin, 24, rue du Faubourg-Saint-Jacques. 75014 Paris. France. 